\title{
A NEURAL NETWORK BASED SMART BUILDING MONITORING SYSTEM USING WIRELESS SENSOR NETWORK
}

\author{
Pradeep K G M ${ }^{1}$, Dr. S.VENKATESAN ${ }^{2}$, Dr.Soundara Rajan ${ }^{3}$ \\ ${ }^{1}$ Research Scholar, Department of Electronics \& Communication Engineering, School of Engineering, \\ Sri Satya Sai University of Technology and Medical Sciences, Sehore Bhopal-466001, Madhya Pradesh \\ ${ }^{2}$ Associate Professor, Department of CSE, Aurora's Technological and Research Institute, Hyderabad. \\ ${ }^{3}$ Dean (Research \& Development), Teegala Krishna Reddy Engineering College, Hyderabad - 500097, Telangana
}

\begin{abstract}
A Smart building monitoring System uses Sensors like temperature Sensor, humidity sensor, motion / occupancy Sensor, contact sensor, Gas/air quality Sensor, Strain Sensor, electrical current monitoring Sensor etc for monitoring an environmental factors and to control devices such as air conditioner, ventilator, alarm, Security grand system etc using sensor information. Our proposed system uses effective neural network to gather information from different Sensor and consolidate it by Information Isolation Node (IIN) and send to sink node The Sink node sends information. to cloud where remote monitoring is done and it also receives Control information from cloud and operates different equipments of building. For efficient implementation only needed information are sent to cloud and necessary action are taken by remote system based on consolidated information sent by Information Isolation Node (IN) through Sink node The Sink node sends information to cloud where remote monitoring is done and it also receives Control information from cloud and operates different equipments of building.
\end{abstract}

Keyword: Smart Building Monitoring system, Information Isolation Node(IIN),Sink Node, Neural Networks

\section{Introduction}

\subsection{Structural Health Monitoring:-}

The Structural Health Monitoring (SHM) is defined as a process of implementing a damage detection and characterization of engineering structures strategy. The SHM process involves the analysis of a system by using the parameters such as: time using periodically sampled dynamic response, amusements from an array of sensors, the extraction of damage sensitive headlining from these assessments, and the analytical investigation of these features to determine the current state of system health. In the long term SHM, the output of the process periodically updates the information regarding the capacity of the construction to perform its motivated function in light of the inevitable aging and degradation resulting from operational environments. After the events that are taken place such as earthquakes or blast loading, SHM is used for rapid condition screening and developing in an objective to provide it in near real time with reliable information regarding the integrity of the structure.

Therefore, the advantage of the SHM is enhancing day by day. A structural health monitoring system is invented, achieved and attempted using Wireless Sensor Networks .Ambient vibration of the structure is audited and is implemented to identify the health status of the structure. With WSN, low cost monitoring is possible without intruding with the action of the structure.

\subsection{NEURAL NETWORK}

The neural network is designed by neural is our brain formats. It collects the information and isolates the data then it send to cluster head.

Two types:

Fixed network

Adaptive network

\subsubsection{Neural Network}

Many neural networks are used in the field of prediction because of their accuracy and effectiveness compared to other methods used. In the method proposed in our paper we use a neural network that is trained under supervision. This neural network is trained using the particle swarm optimization(PSO) algorithm. The network consists of three layers, which are input, output, and hidden. In the input layer we use 10 previous inputs for a network. In the hidden layer we use 12 a hidden node consisting of the sigmoid activation function[17] as in the equation (5). As for the output layer, we use the equation of the linear function. The data used is divided into training and testing 


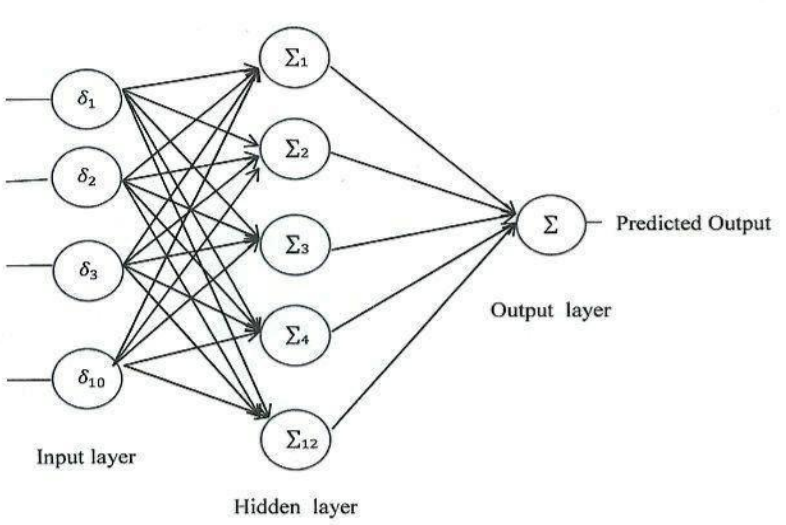

Fig (1) Neural Network Forecasting

$\nabla(v)=\frac{1}{1+\exp (-a v)}$

where (a) is a real constant associated with the function slope in its inflection point. The slope parameters of the $\mathrm{x}$ function (usually given a value of +1 ) are in the term. And when the parameters of the $\mathrm{x}$ function are close to the termination in this case it becomes like a threshold function. The threshold imposes values of either 0 or 1 while the sigmoid function imposes a continuous range of values between 0 to 1 logistical, and 1 to 1 for the hyperbolic tangent function. It can also be observed that the function of sigmoid is different, while the opposite is, the threshold function is defined as either 0 or 1 Then the data were divided into (60\%) training and (40\%) testing, After training the proposed model, its value is measured using square root of mean squared error (MSE) Eq. (6). Where ${ }^{t}$ and $O^{t t}$ are the actual and predicted workloads $M S E=\frac{\sum_{i=1}^{N} \frac{\left(O^{t}-O^{t t}\right)^{2}}{N}}{N}$

\subsubsection{Downsides and difficulties of utilizing wired SHM frameworks:}

1.Huge expense of wiring and instrumentation.

2.Difficulties in conveying and keeping up wiring plant.

3.Difficulties in the executing the huge measure of information.

4.Monitoring the structural building environment is determined by wired frameworks.

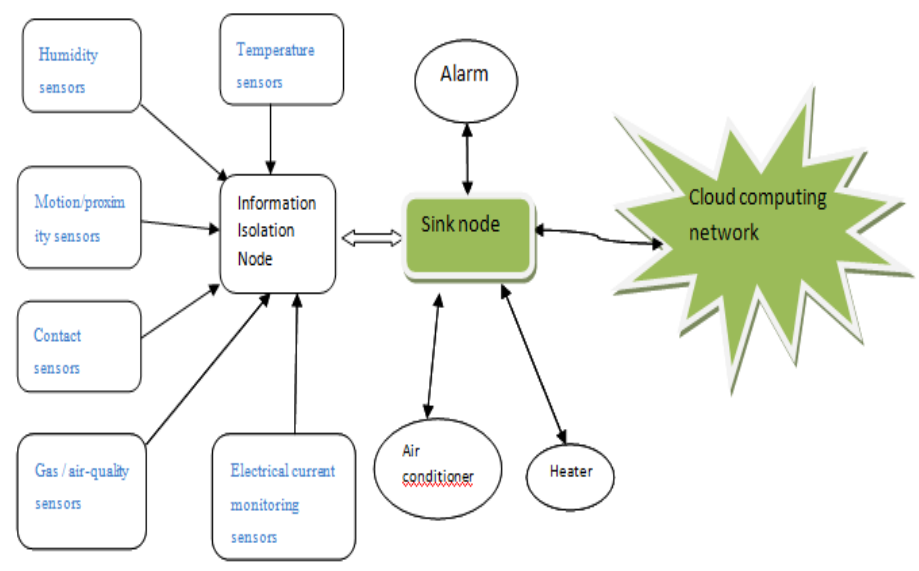

Figure 2 Block Diagram of Structural Health Monitoring System

\subsubsection{Remote Sensor Network}

WSN-based SHM framework offers the potential for minimal effort and solid SHM utilizing a dense array of wireless sensors.

\subsubsection{Favorable circumstances of utilizing remote SHM frameworks:}

1.Low expense and simple establishment and organization,

2.Higher unwavering quality than wired framework,

3.More adaptability for different kind of structures,

4.On-board computational capacity...

\subsubsection{Purposes behind in adapting the Structural building} Monitoring framework:-

1.Damages are the primary cause of structural failure and may happen at various pieces of a structure.

2.Damage location at beginning phase can upgrade the lifetime of the structure .it keep it from abrupt breakdown and sudden collapse.

3.Application and arrangement of SHM frameworks can be helpful for building proprietors, network and governments.

4.Wireless Sensor Networks (WSN) can make a minimal effort, low-cost, reliable, and automated SHM framework can be feasible.

\subsubsection{Necessities for Wireless senor Network organization:}

1.Hardware

2.Software

Hardware Equipment Design of WSU:

2.1.Wireless Sensor Detecting Interface

It Incorporates sensors and simple to-advanced converter (ADC) and to convert analogue sensor signal to digital formats designs

\subsection{Processing Core}


Two important primary parts of wireless remote sensor network are microcontrollers and memory for sensor information

1.Wireless sensor network Remote Radio

2.Wireless Remote radio to send and receive the information different remote sensors.

\subsection{Programming plan of the WSU:}

Embedded programming is carried out on senor

node hubs:

Network Organization topology geography,

Information collecting and gathering,

Information handling calculations,

\subsection{Power management for operating the Circuit board.}

PC have programming for managing with the organization and sensor detected information.

A kind of framework that gives data about any harm happening in the structure.

\subsection{Fundamental elements of Structural building checking:}

- Monitor and evaluate load conditions .

-Examine current plan.

- Verify new systematic strategies and PC computer simulations

- Assess basic structural performance and identify harm in the building parts

- Facilitate assessment and building support works

- Help authority to make quick and right decision in emergency cases

The different types of sensors and they role in maintaining normal environment within a industrial building using actuators are given below

\subsection{Temperature sensors}

It measure heat in building and detect changes in temperature. The machine used in computing and manufacturing is sensible to raise in temperature and these components must be saved from overheating. The room temperature has to be maintained. Any variation we have to send information to Information Isolation Node (IIN). Then from IIN node information send to cloud through the sink node and control information from cloud is received to turn on heater when measured temperature is less than room temperature or turn on air conditioner when measured temperature is greater than room temperature

\section{IF ((measTemp < roomTemp) OR (measTemp > roomTemp)) \\ \{}

Send measTemp to Information Isolation Node (IIN) Trigger on Heater or Air Conditioner via cloud computi

\}

\section{Solid State Gas Sensors For Air Pollution}

Gas sensors for detecting air pollutants have to be able to operate stably beneath deleterious situations, which includes chemical and/or thermal assault. Therefore, strongstate fuel sensors appear like the most suitable in terms of their sensible robustness. The sensors used for detecting air pollution are generally produced surely by means of coating a sensing (metal oxide) layer and a substrate with two electrodes. Typical materials are tin oxide $(\mathrm{SnO} 2)$, zinc oxide $(\mathrm{ZnO})$, titanium oxide ( $\mathrm{TiO} 2)$ and tungsten oxide (WO3) with typical running temperatures of 200 to $400^{\circ} \mathrm{C}$.
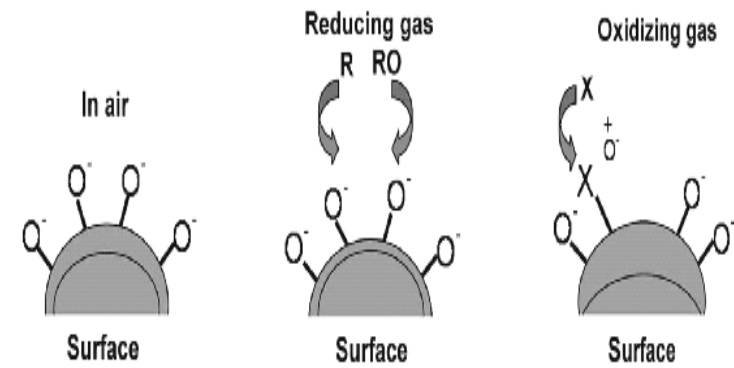

figure3 explains how metal oxide semiconductor detects the pollutant gases.

The depletion area at the surface of steel oxide sensor is due to the absorption of atmospheric oxygen. When the metal oxide sensor absorbs a reducing fuel $(\mathrm{CO}, \mathrm{H} 2)$, depletion area at the surface will be reduced, that means increasing conductivity. On the other hand, if a steel oxide sensor absorbs an oxidizing fuel (NO2), the depletion zone at the surface will be improved, meaning reducing conductivity. In end, an exchange of conductivity/resistance is related to fuel attention. In the case of a $\mathrm{ZnO}$ sensor, the conductivity decreases that means the resistance will increase while the sensor absorbs NOx, dependent on NOx attention.

\section{7 .Nano Sensor Statistical Analysis}

RSM modeling combining with three-level BBD design was used in this experiment to validate its statistical analysis. Box Behnken Design is a self-determining quadratic design which needs fewer combinations of the variables to estimate a potentially complex response function than a full factorial design. In this analysis, BBD with three independent variables (x1: Temperature, x2: Particle fraction (\% Vol), x3: Solution $\mathrm{pH})$ at three-levels was performed. 
$X_{\mathrm{i}}=\left(X_{\mathrm{i}}-X_{0}\right) / \Delta X$

Table 3. Coded and Actual Variables using BoxBehnken Design

\begin{tabular}{|c|c|c|c|c|}
\hline \multirow{3}{*}{$\begin{array}{l}\text { Independent } \\
\text { variables }\end{array}$} & \multirow{3}{*}{ Symbol } & \multicolumn{3}{|c|}{$\begin{array}{l}\text { Coded and actual } \\
\text { level }\end{array}$} \\
\hline & & Low & Center & High \\
\hline & & -1 & 0 & 1 \\
\hline $\begin{array}{l}\text { Temperature } \\
\text { or) }\end{array}$ & $\mathrm{X}_{1}$ & 30 & 45 & 60 \\
\hline Particle Fraction & $X_{2}$ & 1.5 & 3 & 4.5 \\
\hline Solution $\mathrm{pH}$ & $\mathrm{X}_{3}$ & 4 & 8 & 12 \\
\hline
\end{tabular}

Where $\mathrm{Xi}$ is a coded value of the variable, $\mathrm{xi}$ is the actual value of the variable, and $\mathrm{X} 0$ is the actual value of the ith test variable at the center point. The range and levels of individual variables are presented in Table 1. The whole experiment design consisting of 17 experimental runs is shown in Table 2. In the first step of RSM, a proper approximation is implemented to find the relationship between the dependent variable and the set of independent variables.

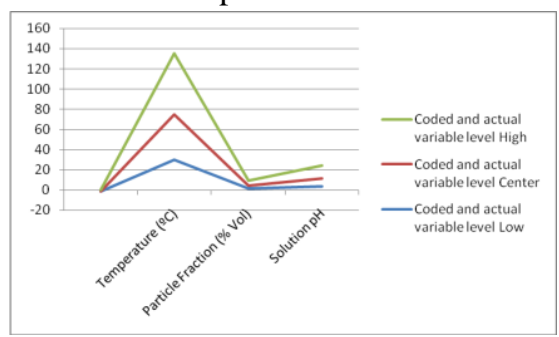

Fig 4 Coded and Actual Variables using BoxBehnken Design line chart Representation.

Table 2 . Experimental data

\begin{tabular}{|l|l|l|l|l|l|l|}
\hline Std & Run & A & B & C & \multicolumn{2}{|l|}{ Thermal Conductivity } \\
& & & & $\mathrm{SnO}_{2}$ with EG/Water $)$ \\
\hline & & & & & Experimental & Predicted \\
\hline 1 & 3 & 45 & 1.5 & 12 & 0.7921 & 0.7920 \\
\hline 2 & 7 & 45 & 3 & 8 & 0.7914 & 0.7914 \\
\hline 3 & 16 & 30 & 4.5 & 8 & 0.7924 & 0.7925 \\
\hline 4 & 18 & 45 & 4.5 & 12 & 0.7931 & 0.7930 \\
\hline 5 & 9 & 60 & 3 & 12 & 0.7920 & 0.7921 \\
\hline 6 & 14 & 60 & 3 & 4 & 0.7897 & 0.7897 \\
\hline 7 & 5 & 30 & 3 & 12 & 0.7899 & 0.7897 \\
\hline
\end{tabular}

\begin{tabular}{|l|l|l|l|l|l|l|}
\hline 8 & 15 & 30 & 1.5 & 8 & 0.7859 & 0.7859 \\
\hline 9 & 11 & 45 & 1.5 & 4 & 0.7909 & 0.7910 \\
\hline 10 & 19 & 60 & 1.5 & 8 & 0.7928 & 0.7928 \\
\hline 11 & 20 & 45 & 3 & 8 & 0.7915 & 0.7916 \\
\hline 12 & 10 & 60 & 4.5 & 8 & 0.7949 & 0.7948 \\
\hline 13 & 17 & 45 & 3 & 8 & 0.7915 & 0.7915 \\
\hline
\end{tabular}

\subsection{Humidity sensors}

It is defined as the amount of moisture content present in the air. When moisture content raises in the air will leads condensation, that makes machines to suffer corrosion. When moisture raises beyond allowable threshold value send information to Information Isolation Node (IIN). Then from IIN node information send to cloud through the sink node and control information from cloud is received to turn on heater and ventilator

IF ((measHumidity > normHumidity $)$ ) \{

Send measHumidity to Information Isolation Node (IIN) Trigger on Heater and Ventilator via cloud computing device \}

\subsection{Motion / occupancy/proximity sensors}

Motion detection can used to secure industry from intruders. This sensor is also useful to operate building controls without manual efforts to perform heating and lighting depending on situation. It also help to decrease both energy usage and operational costs. Occupancy sensors allow company to know which area is used more, or know which shelves or rooms are free at any time. In a huge company, the available areas are effectively used and it reduces the cost. The passive infrared (PIR) motion sensors emits passive infrared rays to detect movement of things and Proximity sensors will shows presence of an things and also the distance between things and these information are send to Information Isolation Node (IIN).Then from IIN node information send to cloud through the sink node and control information from cloud is received to turn on lights, alarm or give information to security guards. IF (Motion Detected)

\section{\{ \\ \}}

\section{Send Motion INFO to Information Isolation Node (IIN) Trigger on Lights and/or Alarm via cloud computing device}

\subsection{Contact sensors}

It is also referred as position or status sensors. This sensor can detect either a door or window is open or close or else it is broken. These sensors are manufactured as pair - one is attached to the door lock or window lock and another attached to the frame side. It uses magnetic field to detect whether the door and frame are attached or detached .These information 
are send to Information Isolation Node (IIN).Then from IIN node information send to cloud through the sink node and control information from cloud is send security guards

\section{IF (Door or Window Disturbed)}

\{

\section{Send INFO to Information Isolation Node (IIN)}

\section{Trigger on Security Guards via cloud computing deviceq Water quality sensors}

$$
\text { \} }
$$

\subsection{Gas / air-quality sensors}

It used to detect various types of gases present in air and also the quality of air is examined. The toxic gases in air can be detected and the hazardous gases in industries are also detected. The presence of $\mathrm{CO}_{2}, \mathrm{CO}, \mathrm{H}, \mathrm{NO}, \mathrm{O}_{2}$, in air are measured. Anything goes abnormal send information to Information Isolation Node (IIN). Then from IIN node information send to cloud through the sink node and control information from cloud is received to turn on alarm. IF (Air polluted)

\{

Send INFO to Information Isolation Node (IIN)

Trigger on Alarm via cloud computing device

2.12 Electrical current monitoring sensors

These sensors practically measure energy usage in any circuit or in any electronic equipment. This is very useful in energy savings the usage of electricity by various components are measured and analysis may be performed whether it is useful or not. The automatic switch off of equipment when not in use is also performed. Any abnormal current usage is send information to Information Isolation Node (IIN).Then from IIN node information send to cloud through the sink node and control information from cloud is received to Switch off circuitry devices.

\section{IF (current fluctuation)}

$$
\{
$$

Send INFO to Information Isolation Node (IIN) Trigger off circuitry board via cloud computing dev

\}

\section{Pressure sensors}

This sensor is used to measure any variation from the pressure standard value. This sensors are used water plant system and also in heating machines.

\section{IF (deviate from standard Pressure) \\ \{}

Send INFO to Information Isolation Node (IIN)

Trigger on Alarm via cloud computing device

\}

It is used to measure alkaline content ,pH values and other organic and inorganic content present in water

\section{IF (deviate from standard water quality)}

\{

Send INFO to Information Isolation Node (IIN) Trigger on Alarm via cloud computing device \}

\subsection{Gas sensors}

It is used to measure air pollution ie how much of other particle or other gases are present in gas

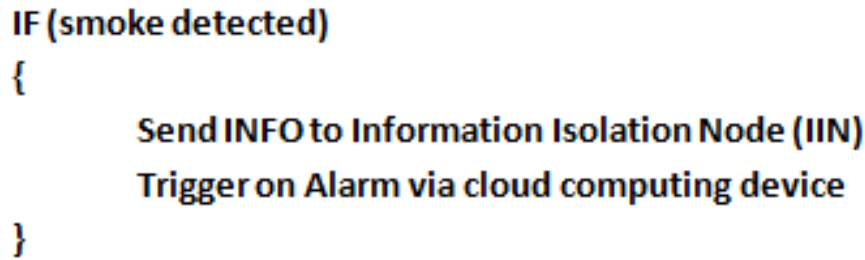

\subsection{Level sensors}

It is used to measure the water, petroleum or other liquid levels in a container. Sea level or dam level can also be measured .Overflow can be avoided by automatic switch using this level sensor.

\section{IF (level raises Standard level)}

\{ \}

Send INFO to Information Isolation Node (IIN) Trigger on Alarm via cloud computing device

2.13 Accelerometer sensors

This sensor is used to detect vibration on any component or iffings. This types of sensor are used in smart phones, industries, vehicles electronic equipments etc.

\section{IF (Vibration detected)}

\{

Send INFO to Information Isolation Node (IIN) Trigger on Alarm via cloud computing device \}

2.14 Carbon Sensors

CNT spatial detecting skins: Using CNT (for example mixture glass-fiber composite) joined to Small solid pillars framed a consistent conductive skin (layer in structure) inside the metal rods. 
Advantages of Carbon Sensors:-

- A direct methods for estimating the appropriated strain fields.

High Sensitivity and Accuracy to recognize the presence, area and seriousness of structural breaks.

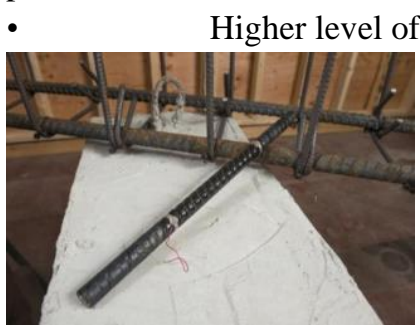

IF (Vibration detected)

\{

\section{Send INFO to Information Isolation Node (IIN) Trigger on Alarm via cloud computing device}

\}

The information isolation node aggregates these sensory information and send to sink node. The sink node convey these information to remote monitoring system via cloud and receives necessary control information from cloud and activates necessary devices in buildings.

The natural disaster is also deducted and efficiently recovery process and alert can be done. Consider the following building infrastructure. A remote sensor network is proposed for observing structures to evaluate the natural disasters like earth quake and tsunami. The sensor hubs utilize exceptionally created capacitive micro electro-mechanical frameworks . They record during a seismic tremor function utilizing a blend of the local a speeding up information and far off setting off from the base station dependent on the quickening information from numerous sensors over the structure.

Structures can dynamically a mass harm during their operational lifetime, because of seismic functions, unanticipated establishment settlement, material maturing, plan mistake, and so forth Intermittent observing of the structure for such harm is along these lines a key advance in normally arranging the support expected to ensure a satisfactory degree of security and administration capacity. In any case, all together for the establishment of a forever introduced detecting framework in structures to be financially.

\section{Sensor Module Architecture}

The square graph of the SHM modules sensor is shown in fig 5. Here the stress and the Acceleration detecting variations of the module utilize a similar center parts. For establishment into the structure these segments are put into a norm off-therack plastic packaging that can be advantageously placed on the divider or roof utilizing screws, and offering access for irregular battery substitution if necessary.

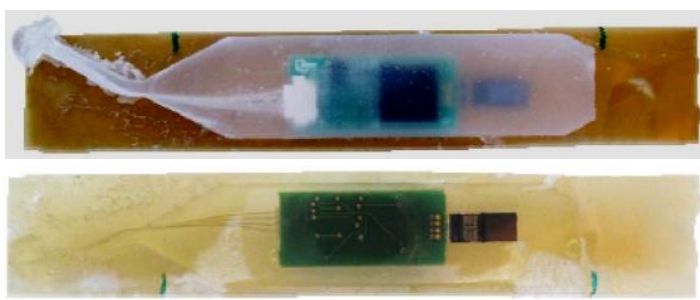

Fig 5 Strain sensor front-end module on polyimide carrier

\subsection{Strain Sensing Front-End Module}

strain sensor is bundled along with the readout ASIC into an extraordinary front-end strain detecting module (Fig. 6) which is installed inside the fortified cement onto the strengthening bar, ideally preceding the pouring of the solid. As appeared in Fig. 6, the sensor is mounted on a polyimide transporter which thus is stuck onto the strengthening bar. A variation of this bundle exists in which the transporter is slight steel, which offers the extra opportunities for welding the transporter to the fortifying bar. The module is formed in PDMS silicone to shield the parts from the climate during establishment and pouring of cement, while staying a precisely agreeable bundle to abstain from misshaping the strain sensor estimation. This front-end strain detecting module is associated with the remainder of the module through a little 4-wire link with a most extreme length of $1.5 \mathrm{~m}$.
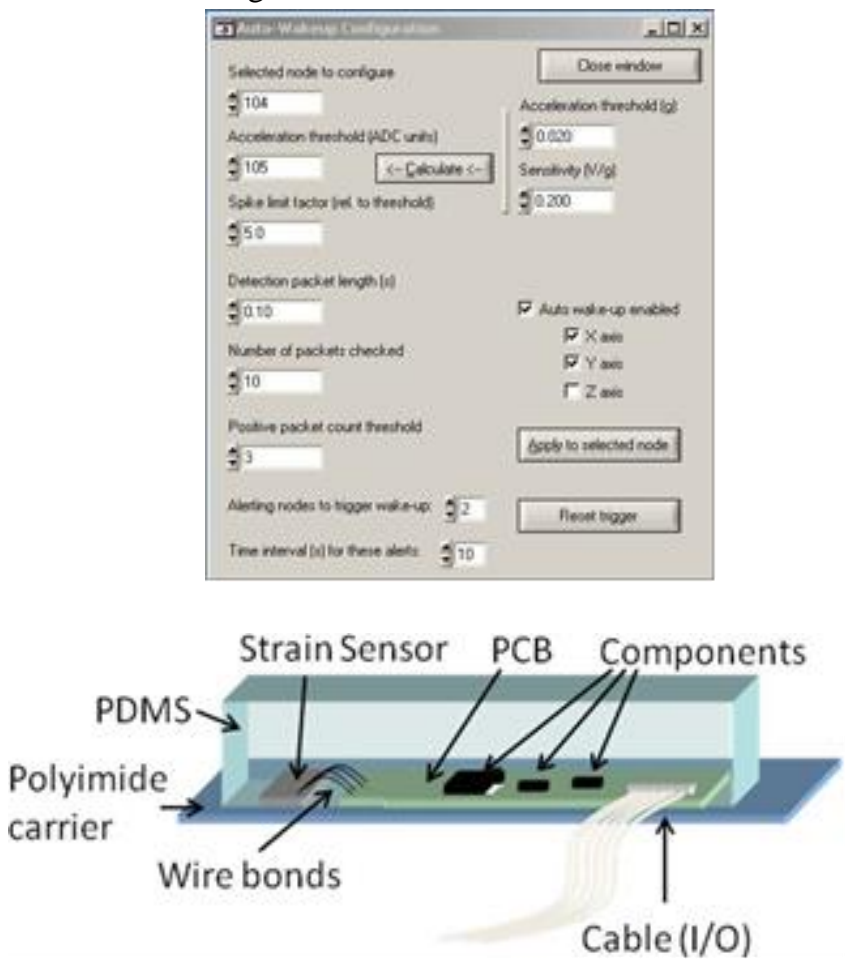

Figure 6 strain sensor Frond end module 


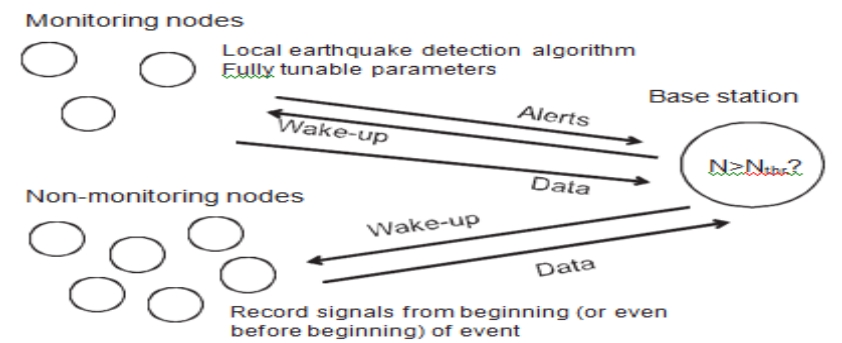

\subsection{POWER CONSUMPTION:}

In our proposed building monitoring sensor, even though the number of sensor used in building is increased, the power consumption is not raised because we are activating few sensor at initial stage for gathering information then whenever there is a need ie situation goes abnormal then only additional sensor are actuated thereby it reduces power consumtion. The simulation result are shown below.

\begin{tabular}{|r|r|}
\hline \multicolumn{2}{|c|}{ SMART BUILDING SENSORS } \\
\hline $\begin{array}{r}\text { INFORMATION } \\
\text { GATHERING }\end{array}$ & $\begin{array}{l}\text { NUMBER OF } \\
\text { SENSOR }\end{array}$ \\
\hline 20 & 5 \\
\hline 33 & 10 \\
\hline 42 & 15 \\
\hline 56 & 20 \\
\hline 60 & 25 \\
\hline 69 & 30 \\
\hline 73 & 35 \\
\hline 80 & 40 \\
\hline 86 & 45 \\
\hline 89 & 50 \\
\hline
\end{tabular}

Table 3 Smart Building Sensor Values

Table 3 Smart Building Sensor Values 3.4 PERFORMANCE MEASURE

Since workload of sink node is reduced by the information isolation node sink node quickly transfer information to

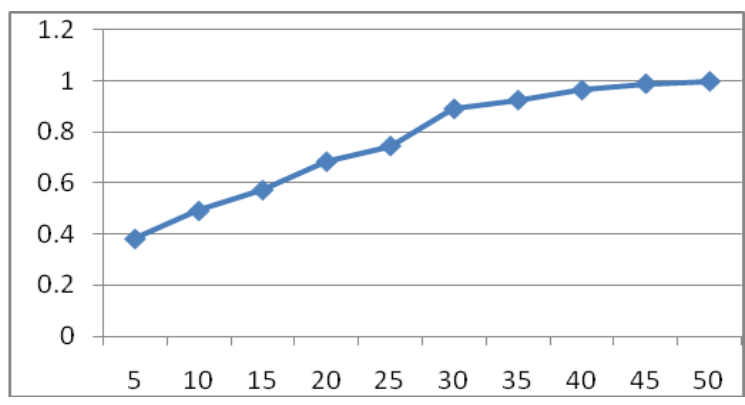

Fig 7 Power Consumption

3.3 INFORMATION GATHERING RATE :

In building 9600 square feet, we increase the number of sensor and gather the information gathering rate. A special node information isolation node is used for gathering information and this node avoid duplicate information ie same information send by different nodes and forward to sink node.A normal scenario as number of sensor increased the information gathering rate is also increased and the simulation result shows approximately 90 percent of information gathering can be done by fixing around 50 sensor nodes within the building

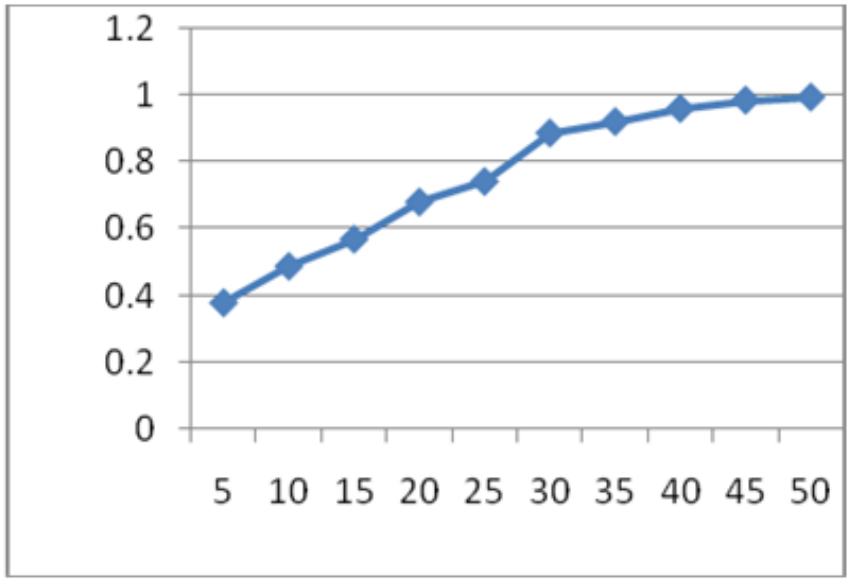

Figure 8 plot for Information Gathering Rate

remote cloud and actuators like alarm, heater, air conditioner are quickly activated based on the information sent from remote cloud. Thus simulation result shows that performance increases as the number of nodes increases 


\begin{tabular}{|r|r|}
\hline \multicolumn{2}{|c|}{ SMART BUILDING SENSOR } \\
\hline & \multicolumn{2}{l|}{ NUMBER OF } \\
PERFOMANCE & SENSOR \\
\hline 0.382 & 5 \\
\hline 0.491 & 10 \\
\hline 0.572 & 15 \\
\hline 0.683 & 20 \\
\hline 0.744 & 25 \\
\hline 0.889 & 30 \\
\hline 0.923 & 35 \\
\hline 0.962 & 40 \\
\hline 0.987 & 45 \\
\hline 0.996 & 50 \\
\hline
\end{tabular}

\section{Table 4 performance characteristics}

\section{Conclusions:-}

Thus various types of building sensors have been placed and the current situation of the building is monitored. If there are any variations in normal conditions then the information is send to remote system through cloud and corresponding activators are activated from the remote system in order to bring the situation to normal condition. This ensures a safety measures for industries and also working employees.

\section{REFERENCES}

[1]. Akyildiz IF, Melodia T, Chowdhury KR (2007) A survey on wire- less multimedia sensor networks. Computer Networks (Elsevier) J 51:921-960

[2]. Al-Turjman F, Alfagih A, and Hassanein H (2012) A novel cost- effective architecture and deployment strategy for integrated RFID and WSN systems. In Proc. of the IEEE Int.Conf. On computing, networking and communications (ICNC), Maui, Hawaii, pp 835- 839

[3]. Al-Turjman F (2016) Impact of user's habits on smartphones' sen- sors: an overview. HONET-ICT International IEEE Symposium

[4]. Al-Turjman F (2016) Hybrid approach for mobile couriers election in smart-cities. In Proc. of the IEEE Local Computer Networks (LCN), Dubai, UAE, pp 507-510

[5]. Soro S, Heinzelman W (July 2009) Cluster head election tech- niques for coverage preservation in wireless sensor networks. Ad Hoc Netw 7(5):955972

[6]. Abdulqader M, Walid A, Khaled A, Abdulrahman A (2016) A robust harmony search algorithm based

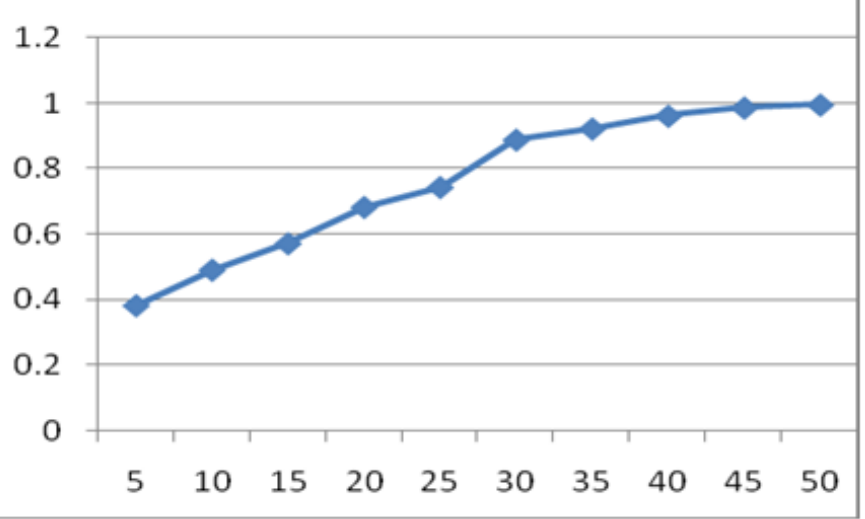

Figure 9 performance measure
Markov model for node deployment in hybrid wireless sensor network. Int $\mathbf{J}$ Geomate 11(27):2747-2754

[7]. Yick J, Mukherjee B, Ghosa D (2008) Wireless sensor network survey. Comput Netw 52:22922330

[8]. Dhawan H, Waraich S (2014) A comparative study on LEACH routing protocol and its variants in wireless sensor networks: a survey. Int J Comput Appl 95(8):975-8887

[9]. Barai LY, Gaikwad MA (2014) Performance evaluation of LEACH protocol for wireless sensor network. International Journal of Innovative Research in Advanced Engineering (IJIRAE) 1(6)

[10]. Mehndiratta N, Manju, Bedi H (2013) Wireless sensor network LEACH protocol: a survey. International Journal of Emerging Research in Management \&Technology ISSN: 22789359(Volume-2, Issue-3)

[11]. Malik M, Singh Y, Arora A (2013) Analysis of LEACH protocol in wireless sensor network. Volume 3, Issue 2

[12]. Heinzelman WR, Chandrakasan A, Balakrishnan H (2000) Energy- efficient communication protocol for wireless microsensor net- works. Proceedings of the 33rd Hawaii International Conference on System Sciences 